\title{
Educación para la transformación social: la propuesta pedagógica de Paulo Freire y el contexto universitario
}

\section{Education as a vehicle for social transformation: Paulo Freire's pedagogy in higher education contexts}

\author{
Ana Gabriela Bell Jiménez ${ }^{1}$ \\ Universidad Estatal a Distancia Costa Rica
}

\begin{abstract}
Resumen. La pedagogía de Paulo Freire busca gestar una educación para la transformación social. Este ensayo argumentativo tiene por objetivos: 1) profundizar en los conceptos pedagógicos implícitos y explícitos en pedagogía de Paulo Freire y su relevancia en vista a la realidad social actual; 2) exponer consideraciones de la implementación de la pedagogía de Paulo Freire en estadios universitarios; 3) argumentar que la implementación de la pedagogía de Paulo Freire en contextos universitarios podría transformar la sociedad. La metodología de construcción del artículo constó de una rigurosa revisión de textos científicos y académicos a la luz de esta temática. Además, se entrevistó a un investigador y autor en el área de la educación dialógica. Se concluye que la labor docente universitaria tiene el potencial de transformar la sociedad en cuanto el docente universitario permita diálogo en su práctica. Se invita a la reflexión y autoevaluación seria del docente universitario y a la institución universitaria en vista de la transformación social que nace de la coherencia entre la teoría que se profesa y la labor docente.
\end{abstract}

Palabras clave. Paulo Freire, educación para la transformación social, diálogo en educación, papel del docente, pedagogía universitaria.

\begin{abstract}
TPaulo Freire's pedagogy views education as capable of achieving social transformation. The objectives of this argumentative essay are: 1) to explain themed-concepts central to Paulo Freire's pedagogy: 2) to establish considerations related to Paulo Freire's pedagogy in higher education settings; 3) to argue that the implementation of Paulo Freire's pedagogy in higher education settings could transform society. A rigorous revision of scientific and academic texts served as foundation for the construction of this essay. An interview with an expert was also conducted. Finally, the conclusions suggest higher education settings have the potential to transform society as long as the university professor allows dialogue in his/her practice. Furthermore, the conclusion is a call for higher education professors and institutions, to engage in a process of self-evaluation in view of the social transformation that rises from the cohesion between the theory professed and the teaching practice.
\end{abstract}

Keywords. Paulo Freire, education for social transformation, dialogue in education, the role of the teacher, higher education pedagogy.

${ }^{1}$ Ana Gabriela Bell Jiménez, Madison Metropolitan School District, Madison, Wisconsin, Estados Unidos. Cursa el programa de doctorado en Educación, en la Universidad Estatal a Distancia, Costa Rica. Dirección electrónica: agbelljimene@madison.k12.wi.us 


\section{Introducción}

La pedagogía de Paulo Freire busca gestar una educación para la transformación social derrocando dinámicas de poder de los opresores a los oprimidos. Esta posición pedagógica se caracteriza por ser política en sus procesos y sus fines. El docente progresista freiriano juega un papel importante en la facilitación de procesos educativos dialógicos de concienciación como praxis transformadora del individuo y de su contexto social (Freire, 1970, 1982, 2002, 2004, 2005, 2010).

El adjetivo "progresista" utilizado por Freire para caracterizar al docente ha sido fuente de crítica y confusión; este refiere el término "progresista" como un concepto que no está vinculado con el liberalismo ideológico que dicta la religión, el comercio y el progreso como inseparables. Para Freire, el docente es progresista por su labor innovadora, específica a su capacidad de escuchar y de ser parte de un diálogo en el que el docente y el estudiante tienen papeles intercambiables, en donde cada uno aprende del otro y con el otro. El docente progresista de Freire es de suma importancia en la práctica transformadora porque es el agente catalítico. En el ejercicio educativo, el docente que utiliza una pedagogía liberadora es crítico, exigente y coherente en sus reflexiones y acercamientos. El docente progresista eficiente entiende y maneja las diferentes variables (métodos, currículo, contenido, educador y educando) para iniciar la praxis transformadora (Villalobos, 2014).

Ahora bien, la universidad, de acuerdo con Ortega y Gassett (2007), se vislumbra como una alternativa educativa superior que puede recibir una persona en lo referente a disciplinas curriculares como física, biología, historia, sociología, filosofía, entre otras. La universidad es la cuna del profesional, el inicio del docente; esta es, a su vez, inseparable de la ciencia y de la investigación científica. En su normalidad, los docentes se forman en estadios universitarios. De esta realización, que es en la universidad en la que se educa al docente, nace el argumento de este ensayo. ¿Puede el estadio universitario ser el punto de partida en la transformación social si en este se utiliza la pedagogía de Paulo Freire?

Este informe hace un aporte al área de estudio, particularmente, en su potencial de reflexión teórica y práctica para el docente universitario y para la entidad universitaria como tal. La lectura cuidadosa del texto revela la importancia de la coherencia ética entre lo que se dice y lo que se hace desde la docencia, así como del valor de la praxis transformadora del diálogo y de la importanciadelahumildad que permiteigualdad. Finalmente, el informe alienta al docente en formación y al docente practicante a transformar la sociedad de su contexto histórico-social.

\section{Metodología}

Para la elaboración de este ensayo argumentativo, se leyeron y analizaron textos científicos y académicos relacionados con la temática de educación para la transformación social, estadios universitarios y la pedagogía de Paulo Freire. De estos, se seleccionaron aquellos que, exitosamente, discutieran o explicaran los conceptos-temas identificados. Además, se entrevistó al Dr. Escobar Galo, investigador con experiencia en educación dialógica y autor de un ensayo reflexivo en el año 2014 sobre perspectivas pedagógicas, en el que se incluye a Paulo Freire. Bajo esta base teóri$\mathrm{ca}$, se interpretaron, se sintetizaron y se relacionaron las ideas para fundamentar las conclusiones.

I. Conceptos pedagógicos implícitos y explícitos en la obra pedagógica de Paulo Freire y su relevancia en vista a la realidad social actual

El primer apartado tiene como fin profundizar, por medio de análisis y crítica, en tres conceptostemas:1) educación para la transformación social; 2) los procesos de diálogo y concienciación como 
praxis transformadora; 3) el papel del educador; y de esa manera evidenciar su relevancia en la realidad social actual.

La definición de los conceptos ideológicos "educación", "transformación social" y "pedagogía" es un punto de partida pertinente en busca de evidencia de educación para la transformación social por medio de la pedagogía. Estos conceptos, por su diaria utilidad semántica, van perdiendo su significado teórico o se van ajustando a las realidades históricas, geográficas, culturales y lingüísticas en las que se emplean.

De los conceptos por definir, educación es quizáselmás comúnmenteusado.Laeducaciónes un fenómeno histórico-social; una manifestación real y como tal se encuentra inserta en el tiempo y espacio, esto significa que se mueve con la historia y mueve la historia. De esta forma, la educación no es un fenómeno neutro a la dinámica de los intereses de los grupos sociales (Jiménez, 2010). Por lo tanto, la educación tiene consideraciones de índole antropológico, porque el ser humano es fundamental en la tarea educativa, ya que "la educación permite aprender a ser lo que se es" (Barrio, 1998, p. 28).

Ahora bien, la pedagogía es la ciencia de la educación. Se dice del conjunto y convergencia de las ciencias que se interesan por la educación, así como de los saberes educativos tanto prácticos como teóricos, enriquecidos por práctica, reflexión e investigación (Jiménez, 2010). El objetivo de la pedagogía antropológica es el mejoramiento de la tarea educativa (Barrio, 1998, p. 12), así, la educación se define como "humanizadora" y humanística, dado que la persona humaniza por completo lo que hace y lo que conoce.La institucionalización educativa juega un papel básico en la creación, el mantenimiento y la transformación de la sociedad.

En su trabajo "El ciclo de socialización", Harro (2000) propone un marco conceptual para explicar la dinámica del poder entre miembros dominantes y miembros subordinados. Dentro de este marco conceptual, este autor establece la institucionalización educativa como un vehículo para reforzar, sancionar, marginar, premiar, privilegiar y discriminar a los individuos de la sociedad. Según refiere, nacemos y nos criamos en áreas geográficas en las que somos iguales o diferentes a otras personas.

Las diferencias varían en género, etnia, color de piel, lengua materna, edad, religión, orientación sexual o clase socioeconómica. Cada rubro nombrado es parte de una identidad social, por lo que cada uno de ellos conlleva valor, ya sea como norma aceptada por la sociedad o como característica no deseada, subordinada o marginada (Harro, 2000). La implementación de una propuesta pedagógica de transformación social es relevante en vista de la realidad social actual. La UNESCO (2016), en su reporte Education for People and Planet (Educación para las personas y el planeta), dictamina que, siguiendo las tendencias de crecimiento actual, el mundo no logrará universalizar la educación primaria antes del año 2042, por ende, deberá esperar al menos hasta el 2059 y el 2084 para hacerlo con la educación intermedia y la educación secundaria.

La universalización de la educación secundaria, en los países más pobres, podría crear un aumento de $75 \%$ en el ingreso per cápita, con lo que 60 millones de personas podrían salir de la pobreza. Si esta meta se lograra para el año 2030, podría prevenir 50000 muertes a causa de enfermedades o de desastres entre los años 2040 y 2050 (UNESCO, 2016, p. 158).

Centrando la atención en los datos de la UNES$\mathrm{CO}$, y la relación entre nivel educativo, índice de pobreza y de salud que se sugiere, ¿vale entonces la pena considerar una apuesta pedagógica que tenga como fin la transformación social? 
El concepto de educación para la transformación social desde la pedagogía de Paulo Freire. La educación es un "fenómeno histórico social" (Jiménez, 2010). En este, Freire descubre un llamado en el aspecto político cuando en las décadas de los años 1940 y 1950 se le ofrece liderar un plan de alfabetización de adultos. La conexión político-histórica viene en este periodo, en Brasil, donde solamente las personas alfabetizadas podían votar. Desde una perspectiva psicosocial, Freire desarrolló e implementó planes curriculares de alfabetización popular que cumplieron con el objetivo mínimo para lograr el voto y que, a su vez, concienciaron a las personas históricamente oprimidas.

Por medio del proceso alfabetizadorconcienciador, el educando renace capaz de definir su historia y la de su pueblo (Soto \& Bernardini, 2010). Freire argumenta que, desde la conquista, los pueblos y los recursos en América Latina han sido explotados y silenciados. De aquí, el concepto de "cultura del silencio", en la cual grandes sectores de la sociedad pierden la capacidad de expresar o de abogar por sí mismos y por sus intereses. Sobre esta sociedad silenciada, el sistema de desarrollo económico florece para reforzar la opresión de las clases obreras y sus sindicatos (Igelmo, 2013, pp. 23-26).

Tomando en cuenta el contexto históricogeográfico, la pedagogía freiriana se caracteriza por ser "sustantivamente política" (Freire, 2005); y por consiguiente, se atribuye al proceso de transformación social. Desde esta perspectiva, la educación es el proceso que libera a aquellas personas silenciadas y oprimidas por sectores dominantes y opresores de la sociedad (LucioVillegas, 2015, p. 9-20). La dinámica de opresor y oprimido freiriana se fundamenta en la hegemonía gramsciana dogmática marxista que dictaquedentrodecadabloquehistóricosegenera una alianza de clases sociales con sus propias relaciones de dominación y de subordinación, así como sus expresiones mecánicas, materiales, filosóficas e instrumentales (Vivero-Arriagada, 2014, p. 129).

Como resultado de su fundamento teórico (gramsciano), Freire piensa en el oprimido como el campesino o la persona que trabaja las tierras. El oprimido es cualquiera a quien se le niega la libertad, el análisis y los acercamientos pragmáticos a la teoría de Freire; estos elementos/ aspectos han sufrido críticas referentes a los límites de la definición de oprimido.

Primordialmente, de acuerdo con LucioVillegas (2015), se señala/reprocha que Freire nunca menciona las dinámicas de opresión entre géneros. La crítica de opresión entre géneros se fundamenta en las tendencias culturales patriarcales contra la mujer y las niñas, y de cómo estas juegan un papel de dominancia en contextos sociales (Lucio-Villegas, 2015, pp. 9-20).

En esa línea, Kathleen Weiler (1991) analiza los avances del feminismo epistemológico, identificando cómo este movimiento social se beneficia de la pedagogía de Paulo Freire y la enriquece en perspectiva al cuestionar el papel de la autoridad del docente para con la educanda. Enseguida, se critica la ausencia de consideración por las relaciones de opresión hacia grupos que son cultural, étnica o lingüísticamente diversos a la población mayoritaria. Esta situación es de sumaimportancia en los patrones migratorios por motivos socioeconómicos y sociopolíticos que han tenido lugar desde la década de los años 1950 hasta la actualidad (Lucio-Villegas, 2015, p. 14).

Un contraargumento a estas críticas es que la amplitud del término freiriano permite el acogimiento de su teoría en una forma global. Visto de esta forma, la definición de Freire es flexible, característica que le permite vigencia en diferentes bloques históricos y geográficos (Lucio-Villegas, 2015, p. 15). 
La educación, desde la pedagogía de Freire, es una contraoferta a la educación bancaria. Se define por educación bancaria la que compara al educando con una cuenta de banco sin fondos en la cual el docente "deposita" saber. Desde esta metáfora, se asume que el educador es conocedor y está por encima del educando, que no es más que un ignorante. En la educación bancaria, los contenidos educativos son determinados por opresores, con motivos económicos y utilitarios (Freire, 1970, p. 51). La dinámica de sabio y de ignorante entorpece el diálogo, ya que el hablar es del sabio y el escuchar es el quehacer de quien no conoce. La educación de Freire, que es la educación para la libertad, derriba los muros que separan al educador y al educando por medio de un proceso dialógico en el cual ambos se educan simultáneamente.

La educación permite la búsqueda del individuo desde y hacia sí mismo (Freire, 2002). Esta búsqueda es colectiva porque existen otros que desean "ser más" (Freire, 2002, p. 8). El individuo, por medio de la reflexión que conciencia, construye una visión de mundo referente a su contexto y a sus acciones. Este proceso de concienciación individual y colectiva transforma el mundo (Soto \& Bernardini, 2010, p. 295).

A modo de síntesis, esta sección hace hincapié en que la educación freiriana tiene como fin la transformación social hacia la liberación. Por medio del quehacer educativo, dentro de un bloque histórico, se tiene como fin derribar las dinámicas de dominación y de subordinación de las clases sociales. La sociedad freiriana se divide en opresores y oprimidos, estos últimos son parte de una "cultura del silencio" que deshumaniza, es decir, les niega la libertad. Esta definición de oprimido ha sido criticada y expandida para incluir la opresión que existe entre géneros o por diversidad cultural, lingüística, religiosa o étnica.
La siguiente sección desarrolla los conceptos de diálogo y concienciación, procesos mediante los cuales las personas oprimidas encuentran su palabra y crean conciencia para transformar la sociedad.

Los conceptos de diálogo y concienciación. El diálogo es la herramienta que permite la búsqueda del sí mismo y la cooperación hacia la transformación social (Freire, 1970, p. 52). De acuerdo con Soto y Bernardini (2010), para alfabetizar masivamente a adultos en Brasil, Freire desarrolló e implementó la propuesta pedagógica llamada "círculos de cultura", los cuales eran grupos de, aproximadamente, 30 adultos analfabetos que lograban escribir su palabra. Los "círculos de cultura" fueron exitosos por su característica dialógica (Freire, 1970, p. 53).

Mediante el diálogo, los educandos hallan sus palabras. El diálogo es imposible en ausencia de las palabras. Asu vez, estas requieren reflexión que evidencie comprensión y la construcción del mundo de quien las dice. El que escucha la palabra la entiende desde su construcción social. La acción-reflexión de la palabra conlleva a la praxis transformadora. La praxis transformadora conciencia a las personas para la participación del mundo (Freire, 1970). Consecuentemente, la transformación del mundo requiere acciones concretas (Freire, 2005).

Una crítica importante al concepto dialógico de Freire es sobre la naturaleza explícita de la palabra escrita; de esta forma, se excluye la oración o la palabra hablada (Lucio-Villegas, 2015, p. 9). La crítica toma valor en los estudios realizados en América Latina en las décadas de los años 1960 y 1970, que demostraron profunda inequidad en los sistemas de comunicación. Estos estudios propusieron modelos alternativos de comunicación que permitieron no solo la recepción de la información, sino también la creación de comunicación deíndole contestataria, 
con el fin de lograr diálogo entre las clases sociales (Accorssi, Scarparo \& Pizzinaro, 2014, pp. 31-42). Algunos ejemplos delaimportancia dela oralidad y de la comunicación alternativa en respuesta a las experiencias sociales de este bloque social, siguiendo a Accorssi, Scarparo y Pizzinaro (2014), se dan en las radioescuelas colombianas $\mathrm{y}$ en las radios sindicales mineras bolivianas.

La praxis de reflexión y de acción, que emerge de la palabra mediante el diálogo, permite la comprensión del mundo y su lectura. Esta premisa sugiere, entonces, que para lograr comunicación los individuos deben tener algún grado de coincidencia en su lectura del mundo (Freire, 1982). Tres aditivos catalíticos que facilitan la defensa de los derechos son: 1) lectura del mundo respecto a realidad política, 2) movilización-acción y 3) organización revolucionaria (Freire, 1970).

Basado en lo anterior, se discute que la naturaleza dialógica de la educación es una categoría esencialpara la psicología social en su ontología y epistemología. El diálogo necesita del "otro" en la sociedad para la construcción del "yo" utilizando un marco ético. La naturaleza ética del diálogo emana de la identificación del "otro" diferente al "yo" en la construcción de la conversación (Accorssi, Scarparo \& Pizzinaro, 2014, p. 40). Por medio de la praxis transformadora, el oprimido se autolibera y, en su liberación, le da libertad al opresor (Freire, 1970, 1982, 2005).

Una consecuencia del proceso de concienciación es el miedo a la libertad. El miedo a la libertad es la desesperanza que ocasiona que el oprimido perciba que su opresión no tiene salida. Algunas manifestaciones del miedo a la libertad son el temor por perder el trabajo u hogar, circunstancias que pueden paralizar la acción y la praxis transformadora (Freire, 1970, 1982, 2005).
De este apartado, se sintetiza que, de la acciónreflexión de la palabra, nace el proceso de praxis transformadora, la cual permite la educación liberadora. La praxis transformadora, entonces, catapulta el proceso de concienciación del individuo para la sociedad. La concienciación se vale de la palabra y el diálogo, estos a su vez construyen lo que se comprende del mundo. Este proceso en cadena (praxis transformadora) se detiene algunas veces por el miedo a la libertad. El miedo a la libertad imposibilita la acción política revolucionaria. Por lo tanto, entendiendo la importancia de la característica dialógica de la educación, ¿cuál es el papel del docente en la facilitación de educación dialógica desde la pedagogía de Freire?

El papel del educador. Desde su pedagogía, Freire (2005) identifica el hecho de educar como un acto crítico-creador relacionado con las acciones enseñar-aprender mediado por la palabra-diálogo. De este modo, el educador tiene como tarea primordial el respeto a la capacidad crítica del educando. Con este fin, el educador emplea rigurosidad académica para acompañar al educando en la reconstrucción y en la construcción de saberes. El educador tiene la responsabilidad de investigar para, así, extender los límites de su construcción del mundo. La investigación ha de ser acompañada del diálogo y del pensamiento crítico. Se destaca que uno de los valores progresistas más significativo es admitir que enseñar no es la transferencia de conocimientos, sino el acto colaborativo de construcción de conocimiento nacido de la praxis transformadora (Freire, 2004).

En el texto metodológico referencial titulado "Cartas a quien pretendeenseñar", Freire (2010) contextualiza los saberes de la pedagogía del oprimido (Freire, 1970) y la educación como práctica de la libertad (Freire, 1982). En "Cartas a quien pretende enseñar", Freire (2010) explica la teoría (ahora madura) a los educadores y 
sus aplicaciones metodológicas filosóficas con educandos en la niñez, adolescencia y adultez. Las cartas ilustran el papel dialógico de la enseñanza-aprendizaje; en ellas, Freire reconoce la dificultad del papel del educador y le insta a no "paralizarse". Las cartas guían al educador en su facilitación de diálogo con el educando y no al educando (Freire, 2010).

Freire expresa su posición en lo referente a la formación académica universitaria al apuntar que "el papel del orientador no puede ser un programador de la vida intelectual del orientado, estableciendo reglas sobre lo que puedo o no puede escribir" (Freire, 2008, p.185) y que "la Academia no puede ni debe ser un contexto inhibidor de la búsqueda, de la capacidad de pensar, de argumentar, de preguntar, de criticar, de dudar, de ir más allá de los esquemas preestablecidos" (Freire, 2008, p. 187). De esto se infiere que el trabajo liberador universitario tiene como punto de partida el proceso metodológico dialógico de aprendizaje (J.P. Escobar Galo, comunicación personal, 22 de abril, 2017).

El docente progresista freiriano es vital en la ejecución pedagógica. Tener la educación como vía de transformación social, además del diálogo y la concienciación como praxis, no es suficiente para liberar. Dicho de otra forma, sin un docente progresista, no se logran los objetivos de libertad.

II. Consideraciones para la implementación de la pedagogía de Paulo Freire en estadios universitarios

El segundo apartado aborda las recomendaciones de la "Conferencia mundial sobre la educación superior en el cambio social y el desarro1lo". Además, explora los aportes Paulo Freire a la pedagogía universitaria y comparte la propuesta pedagógica de Freire, desde la perspectiva del Dr. Juan Pablo Escobar Galo.

El papel de las universidades como agentes sociales. $\mathrm{La}$ "Conferencia mundial sobre la educación superior" (UNESCO, 2009) les ofreció a los
Estados participantes guías en lo referente al papel de la educación superior para el cambio social y el desarrollo. Del informe general, en el área de responsabilidad social, se rescatan las siguientes citas:

La educación superior debería asumir el liderazgo social en materia de creación de conocimientos de alcance mundial para abordar retos mundiales, entre los que figuran la seguridad alimentaria, el cambio climático, la gestión del agua, el diálogo intercultural, las energías renovables y la salud pública (UNESCO, 2009, p. 2).

La educación superior debe no solo proporcionar competencias sólidas para el mundo de hoy y de mañana, sino contribuir además a la formación de ciudadanos dotados de principios éticos, comprometidos con la construcción de la paz, la defensa de los derechos humanos y los valores de la democracia (UNESCO, 2009, p. 2).

Las citas demuestran la existencia de un marco internacional de desarrollo que afilia la educación superior con la responsabilidad social. Ninguna de las citas tiene una relación directa, exclusiva o de uno-a-uno con los fundamentos freirianos; sin embargo, se puede leer entre líneas la similitud de su espíritu. Asimismo, se puede argumentar que el "diálogo intercultural" referido por la UNESCO (2009) requiere de un diálogo hacia la praxis transformativa de Freire. De igual forma, se defiende la visión de "contribuir además a la formación de ciudadanos dotados de principios éticos, comprometidos con la construcción de la paz, la defensa de los derechos humanos y los valores de la democracia" (UNESCO, 2009, p. 2), la cual es una manifestación del individuo que se cambia a sí mismo y a la sociedad por medio de participación activa expuesta por Freire.

La existencia de un marco internacional de desarrollo es básica en lo referente a la tesis de este ensayo, ya que a pesar de que Paulo Freire marca las pautas teóricas para una 
educación transformativa, nunca ejerce un estudio que concrete estas prácticas explícita o exclusivamente (Igelmo, 2013, p. 13).

Aportes de Paulo Freire a la pedagogía universitaria. Partiendo de una concepción de universidades como entes sociales para la transformación social, Freire ofrece algunas características globales del docente. A pesar de que estas no están diseñadas para los estadios universitarios exclusivamente, la investigadora rescata tres características, a fin de potenciar renovación sistemática.

La primera característica rescatada es la de coherencia ética. En otras palabras, el mensaje que ofrece el docente en su discurso no debe diferir desu actuación. Las acciones y la retórica del docente requieren de un alineamiento de coherencia ética de respeto propio y hacia los educandos. Así, si el docente predica diálogo, debe invitar el diálogo en su quehacer educativo; si el docente exige ampliación de ideas, él mismo debe de ser capaz de extender su visión de mundo. Si la expectativa es tolerancia, debe entonces el docente ser tolerante (Freire, 1982).

La segunda característica es la humildad. Es decir, el docente asume que es humano e incompleto y, por ende, puede y deber ser parte de una praxis transformadora con los educandos. La humildad reconoce que cada uno entiende el mundo desde su perspectiva, que no existe un ser humano incapaz de aprender más; ni tampoco existe quien no sepa nada. La humildad iguala al docente con el educando. En esta igualdad existe respeto (Freire, 1982, 2002, 2004).

Latercera característica esla anuenciaalcambio. El docente deber tener la disposición de cambiar su práctica hacia la praxis transformadora y a la acción política que esta conlleva (Freire, 2006).

De igual forma, Freire (2004) propone saberes indispensables para lograr la práctica educativa. Una vez más, estos no son identificados por Freire como exclusivos para los docentes universitarios, pero la investigadora los resalta por su potencialidad en estos estadios:

- Rigor metodológico que permita el fortalecimiento de la capacidad crítica del educando.

- Investigación en paralelo con la enseñanza. Así, el docente reconstruye su visión del mundo para entender lo desconocido.

- Respeto a los educandos y a sus saberes. El respeto se demuestra en el diálogo y en la discusión relacionada a los contenidos de estudio.

- La autoridad del docente se afianza en cómo este actúa, en su coherencia ética.

De esta sección, se concluye que la pedagogía de Freire aporta significativamente en la filosofía universitaria en la caracterización del docente y en los saberes que este debe tener para facilitar la transformación social.

Propuesta pedagógica freiriana. Caso: entrevista con Juan Pablo Escobar Galo. En su ensayo, Escobar Galo (2014), mediante un texto reflexivo, apunta a la necesidad de ahondar en las diferentes propuestas pedagógicas, con el fin de identificar aquellas que "de alguna u otra forma, cuestionan el sistema educativo tradicional" (p. 85). En una entrevista otorgada a esta investigadora (comunicación personal, 22 de abril de 2017), el Dr. Escobar Galo comparte su construcción personal, pedagógica filosófica de la pedagogía de Paulo Freire en estadios universitarios:

Del tema de libertad y de cómo la libertad de cátedra puede influir en la utilización de prácticas docentes liberadoras, responde: “Creo que el tema de la libertad de cátedra es un tema que apunta por el ejercicio de la libertad en el hacer profesional, pero no es en la libertad de cátedra en donde radica el problema de la educación opresiva, sino en las formas o relaciones opresivas que condicionan 
las relaciones educador y educando", y añade: "Hay que poner más atención al cómo y no tanto al qué implica la libertad de cátedra. El "cómo" implica romper las relaciones de amo-esclavo que establece el modelo bancario" (J.P. Escobar Galo, comunicación personal, 22 de abril, 2017).

Ante la pregunta de la responsabilidad de la universidad en cuanto a la utilización de prácticas pedagógicas antieducativas u opresoras, aprecia que "una formación universitaria que no reconoce el acontecer histórico político de sus integrantes es una universidad no liberadora. Una universidad que pretende dominar por medio de elementos teóricos o metodológicos a sus estudiantes es una universidad opresiva. Una universidad que no propicia el pensamiento crítico y el ejercicio de la autonomía moral es una universidad bancaria" y se debe " poner atención a las prácticas de la vida cotidiana y las formas de administración del poder dentro de la universidad" (J.P. Escobar Galo, comunicación personal, 22 de abril, 2017).

A la interrogante del potencial del estadio universitario como una entidad social para la transformación, apunta que "para lograr la transformación social desde la universidad es necesario deconstruir, la universidad. Uno de los puntos claves es ayudar a los profesores a desaprender los modelos bancarios y reasumir la labor educativa desde la pedagogía crítica; de lo contrario, la educación superior no será liberadora, sino servil al sistema opresor" (J.P. Escobar Galo, comunicación personal, 22 de abril, 2017).

La construcción pedagógica filosófica personal del Dr. Escobar Galo ejemplifica conexiones entre la aplicación práctica de la pedagogía liberadora de Paulo Freire y el estadio universitario actual. De relevancia, y a forma de síntesis, se apunta que las recomendaciones son alcanzables y aplicables para aquellos docentes e instituciones que deseen cocrear transformación so- cial. Las impresiones del Dr. Escobar Galo son alentadoras en su espíritu de esperanza.

\section{Conclusión}

La pedagogía de Paulo Freire se explica en la construcción de tres conceptos-temas:

1) La educación para la transformación social es sociopolítica: tiene como fin concienciar al individuo, para que este reflexione-actúe en su contexto histórico-geográfico por medio de la participación política-activa. Esta educación es un proceso liberador dialógico de quienes han sido silenciados $\mathrm{u}$ oprimidos por sectores opresores de la sociedad.

2) La educación dialógica, acción-reflexión de la palabra, inicia la praxis transformadora y la concienciación. La concientización es un acto personal hacia la sociedad que requiere de la palabra y del diálogo para la construcción de la comprensión propia del mundo. La concientización provee claridad política que insta la acción en contra de los sistemas opresores y logra la organización revolucionaria.

3) El educador progresista trabaja en contra de la educación bancaria y en pro de la pedagogía para la libertad. El educador y el educando coconstruyen una visión de mundo por medio del diálogo fundamentado en el respeto. El docente freiriano progresista respeta la capacidad crítica del educando. Por medio del diálogo, el educador y el educando juegan papeles intercambiables.

Se reconoce que la sociedad del bloque histórico en el que vivimos está regida por dinámicas de opresores y oprimidos. La definición de oprimido ofrecida por Freire es tan flexible que permite que cada docente reflexione en las prácticas sociales de su contexto $y$, en estas, reconozca a quién se ha oprimido. Se conciencia, además, en la importancia del docente hacia la implementación de pedagogía para la libertad y se hace hincapié en este punto, porque el docente puede 
elegir en su práctica personal la utilización del diálogo para la libertad.

Al generarse la formación docente primordialmente en universidades, se apunta hacia las universidades como agentes para el cambio y desarrollo social. La "Conferencia mundial sobre la educación superior" (UNESCO, 2009) comparte intencionalidad con la pedagogía para la libertad en la creación de diálogo intercultural y en la formación de ciudadanos comprometidos con la defensa de los derechos humanos.

La investigadora no encuentra, y por ende no incluye, evidencia de la implementación científica de la pedagogía freiriana en universidades actuales. Aun así, descubre argumentos pedagógicos filosóficos de peso, que sumados a su construcción social, invitan a la implementación de la educación dialógica en el estadio universitario. Este ensayo argumenta que el docente es el punto de conexión entre la educación para la transformación social y el diálogo como praxis transformadora para lograrlo. Sin docentes progresistas, capaces de respetar al educando, no se logra la libertad.

El Dr. Juan Pablo Escobar Galo propone la implementación de pedagogía liberadora; para ello, explica la dualidad de responsabilidad requerida. En primera instancia, la universidad como agente social es responsable de apoyar a los docentes a deconstruir la educación bancaria; y finalmente, el docente tiene la responsabilidad ética y profesional de acompañar al educando, de facilitar praxis transformadora por medio del diálogo. De este modo, la educación transformará la sociedad en cada salón de clase y con cada docente que invite al diálogo, al respeto y a la praxis transformadora.

Ahora bien, la reflexión del Dr. Escobar Galo aclara una confusión común. El docente puede mantener su derecho a libertad de cátedra en la elección de contenidos; pero al mismo tiempo, puede emplear pedagogía liberadora, dialógica para derrocar relaciones de amo-esclavo. La praxis transformadora fundamentada en el diálogo y uso de la palabra puede ser asumida, entonces, como un valor ético y profesional de la educación universitaria como ente social para la transformación social.

La investigadora, desde su construcción del mundo, conoce algunas de las manifestaciones de miedo a la libertad en estadios universitarios, tales como el temor a la represalia de un docente, la humillación pública, la pérdida financiera que implica el perder, el desgaste emocional de un proceso de queja. Quizás, la más terrible de las manifestaciones al miedo que ha experimentado la investigadora es la continuación a la dinámica de amo-esclavo que caracteriza la educación tradicional para quienes ya son parte de grupos etno-lingüísticos históricamente marginados.

La investigadora, inmigrante y persona perteneciente a un grupo etno-lingüístico históricamente marginado y con 19 años en el área de educación para poblaciones marginadas, invita a los docentes universitarios y a las universidades a reflexionar, con seriedad, en su responsabilidad social. Como se explica en los párrafos introductorios de este ensayo, la sociedad actual necesita ser renovada y la educación universitaria puede eficazmente hacer esto. Para lograrlo, lo que sale de la boca del docente respecto a teoría debe ser práctica en sus manos. No sirve de nada aprender de Freire mientras se está de rodillas besándole los pies a un sabio. No es aceptable que un docente humille al que ha tenido la disposición de aprender porque se conoce incompleto. No es moral que se permitan y se defiendan prácticas antieducativas. El educando ha de ser respetado como ser humano que es parte importante de la historia, que tiene saberes, contextos y construcción social. El diálogo y la praxis transformadora han de ser la herramien- 
ta metodológica de excelencia en la universidad; sin ellas, continuaremos en la desesperanza.

De igual forma, los educandos universitarios (docente practicantes o en formación) deben ser capaces de reconocer los sistemas de opresión en las aulas universitarias, de los colegios y de las escuelas. Es imperativo que se controle el miedo a la libertad para ser liberadores de los propios educandos; que, al final de cada jornada laboral, se pueda decir: hoy fui liberado y liberador.

Cada persona en la sociedad puede ser parte de una praxis de reflexión que le permita encontrarse y encontrar al otro. De esa forma, en el ámbito educativo, ¿cómo se utiliza la palabra, para humanizar o para humillar? ¿Se elige ser opresor? ¿Cuál es la reacción ante la búsqueda de libertad de los estudiantes? Cada educador puede preguntarse ¿quiénes y cómo son las personas en condición de oprimidos en el salón de clase? La opresión de la que escribe Freire se puede trasladar a diversos contextos educativos, en cualquier punto del tiempo, incluyendo la actualidad. Esto le ofrece al docente, a la universidad y a la sociedad la oportunidad de participar en reflexión y reconocimiento en el diario vivir para tomar decisiones rutinarias, tales como demostrar interés y respetar la dignidad de cada uno de los estudiantes.

\section{Referencias}

Accorssi, A.; Scarparo, H. \& Pizzinato, A. (septiembre-diciembre, 2014). La dialogicidad como supuesto ontológico y epistemológico en Psicología Social: reflexiones a partir de la Teoría de las Representaciones Sociales y la Pedagogía de la Liberación. Revista de Estudios Sociales, (50), 31-42. doi: 10.7440/ res50.2014.06

Barrio, J. M. (1998). Elementos de Antropología Pedagógica. Madrid: Rialp.

Escobar-Galo, J. P. (mayo, 2014). ¿Educar es generar esperanza o replicar un sistema?
Perspectivas pedagógicas de Freire, Russell e Illich. Revista Calidad en la Educación Superior, 5(1), 84-98.

Freire, P. (1970). Pedagogía del oprimido. México: Siglo XXI Editores. Recuperado de http:// www.servicioskoinonia.org/biblioteca/general/FreirePedagogiadelOprimido.pdf

Freire, P. (1982). La educación como práctica de la libertad. Argentina: Siglo XXI Editores. Recuperado de https://asslliuab.noblogs. org/files/2013/09/freire educaci\%C3\%B3n_ como_pr\%C3\%A1ctica libertad.pdf -1.pdf

Freire, P. (2002). Educación y cambio (5. a ed.). Buenos Aires: Galerna. Recuperado de http://derechoepja.org/wp-content/ uploads/2015/05/freire-paulo educacicion-y-cambio.pdf

Freire, P. (2004). Pedagogía de la autonomía. Sao Paulo, Brasil: Paz e Terra S. A. Recuperado de http://www.bibliotecavirtual.com.ar/libros/filosofia/pedagogia $\% 20 \mathrm{de} \% 201 \mathrm{a} \% 20$ autonomia\%20freire.pdf

Freire, P. (2005). Pedagogía de la esperanza. México: Siglo XXI Editores. Recuperado de http://www.cronicon.net/paginas/Documentos/paq2/No.11.pdf

Freire, P. (2006). El grito manso. Argentina: Siglo XXI Editores.

Freire, P. (2008). Cartas a Cristina. Reflexiones sobre mi vida y trabajo (3. ํ ed.). México: Siglo XXI Editores.

Freire, P. (2010). Cartas a quien pretende enseñar. Argentina: Siglo XXI Editores. Recuperado de http://josemramon.com.ar/ wp-content/uploads/Para-educadores-Paulo-Freire-Cartas-a-Quien-Pretende-Ensenar-2002.pdf

Harro, B. (2000). The cycle of socialization. En M. Adams, W.J. Blumenfeld, R. Castañeda, H.W. Hackman, M.L. Peters, \& X. Zuñiga. 
(eds.). Readings for diversity and social justice: An anthology on racism, antisemitism, sexism, heterosexism, ableism, and classism (15-21). New York: Routledge.

Igelmo, J. (enero-junio, 2013). Paulo Freire y John Holt: de la educación liberadora a la libertad más allá de la educación. Educació $i$ Història: Revista d'Història de l'Educació, (21), 13-35.

Jiménez, K. (2010). Educación, pedagogía, didáctica y currículo. San José, Costa Rica: Universidad de Costa Rica.

Lucio-Villegas, E. (2015). Paulo Freire. La Educación como Instrumento para la Justicia Social. Revista Internacional de Educación para la Justicia Social (RIEJS), 4(1), 9-20.

Ortega y Gasset, J. (2007). Herederos de José Ortega y Gasset. Madrid: Biblioteca Nueva.

Soto, J. A. \& Bernardini, A. (2010). La educación actual y sus fuentes filosóficas (2.. ed.). San José, Costa Rica: EUNED.

United Nations Educational Scientific and Cultural Organization (UNESCO, 2009).
“Conferencia mundial sobre la educación superior-2009: La nueva dinámica de la educación superior y la investigación para el cambio social y el desarrollo". París: Autor.

United Nations Educational Scientific and Cultural Organization (UNESCO, 2016). Education for people and planet: Creating sustainable futures for all (2nd Ed.). París: Autor.

Villalobos, D. C. (enero-junio, 2015, 2014). Planteamientos pedagógicos relacionados con las ciudadanías propias en la pedagogía crítica de Paulo Freire. AGO.USB, 15(1), 195-215.

Vivero-Arriagada, L. A. (2014). Una lectura gramsciana del pensamiento de Paulo Freire. Cinta moebio, (51), 127-136.

Weiler, K. (diciembre, 1991) Freire and a Feminist Pedagogy of Difference. Harvard Educational Review, 61(4), 449-475. doi: 10.17763/ haer.61.4.a102265j168rju84

Recibido: : 27 de junio de 2017 Aceptado: 05 de octubre de 2017 